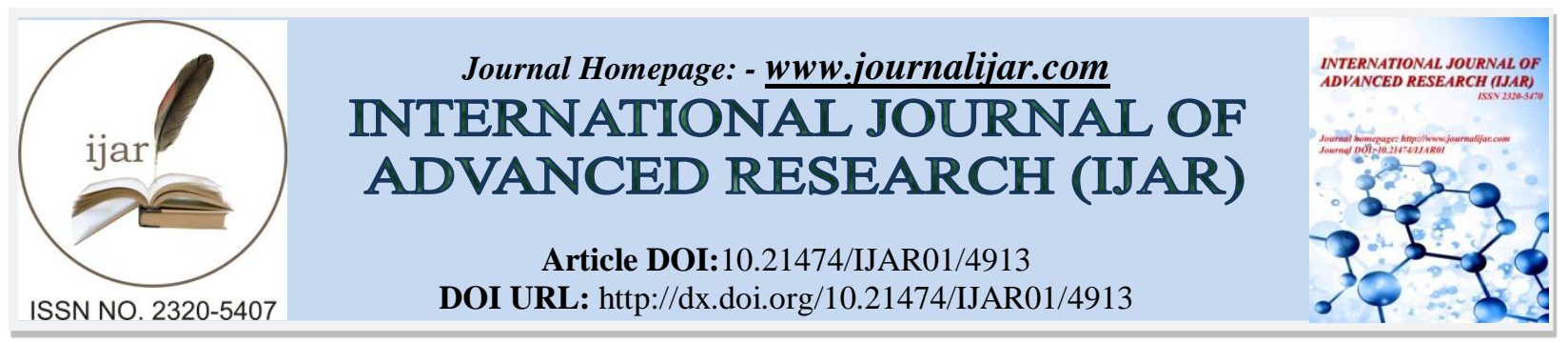

RESEARCH ARTICLE

\title{
A TMT STUDY COMPARING METS AND DOUBLE PRODUCT OF ATHLETIC VERSUS NONATHELETE TEENAGERS OF NORTH-EAST ASSAM.
}

\author{
Dr. Rimpy Bhuyan.
}

\begin{abstract}
Manuscript Info
Manuscript History

Received: 21 May 2017

Final Accepted: 23 June 2017

Published: July 2017
\end{abstract}

Abstract

\section{Introduction:-}

Treadmill test has become a very important clinical investigative tool to assess cardiovascular status of patients in medicine. It has also become very promising in Medical Physiology to know and learn about the effect of exercise on human body.

Aerobic exercises can be defined as any activity that uses large muscle groups; that can be maintained continuously and are rhythmic in nature. The MET concept provides a convenient method to describe the functional capacity or exercise tolerance of an individual as determined from progressive exercise testing. METs (Metabolic Equivalent) help to quantify the energy cost of activities. A MET is defined as the resting metabolic rate, i.e., the amount of oxygen consumed at rest $(3.5 \mathrm{ml} \mathrm{O} 2 / \mathrm{kg} / \mathrm{min}),(1.2 \mathrm{kcal} / \mathrm{min}$ for a $70 \mathrm{~kg}$ man $)$. Thus $2 \mathrm{METs}$ will require twice the resting metabolism and 3 METs will require thrice the resting metabolism. McArdle et al. presented a classification system by which light work elicits an energy expenditure upto 4 METs ( 1 litre of $\mathrm{O} 2$ /min) and heavy work requires 6 to 8 METs. Exercise capacity in METs can be estimated on the basis of the speed and grade of the treadmill. Likewise Double product indicates the myocardial working capacity of the heart; and is estimated by the product of the heart rate and the systolic blood pressure. The normal range is usually between 20,000-25000.Thus METs and double product estimated from a treadmill exercise test can give us an idea about the functional capacity of aerobic exercises among individuals.

\section{Aims \& objective:-}

The present study was undertaken, to compare, the functional capacity of aerobic exercises, in terms of METs and Double product, through treadmill exercise tests, among few teenagers (age 18-20 years) of northeast Assam who play sports, versus those who live a sedentary lifestyle. Such comparative evaluation might prove useful in exercise prescription for different individuals with different lifestyle.

\section{Materials \& methods:-}

1. The subjects for the study were chosen from among the first year MBBS students coming for medical studies to Assam Medical College from different parts of north east Assam during 2014-15.

2. Forty healthy male subjects of age group 18-20 years were included in the study group.

3. Anyone with history of heart disease or any other physical disability was excluded from the study. 
4. A written informed consent was taken from all; And a detail history \& physical examination of each subject was recorded.

5. 20 subjects with an active athletic lifestyle, playing different sports: and 20 subjects with a sedentary lifestyle were considered as two study groups, group A \& group B respectively.

6. The BMI of all the subjects was recorded.

7. The equipment used was a treadmill of mortara with Xcribe system installed, operated by PC based stress exercise testing system.

8. Proper instructions were given to the subjects about the treadmill and then the exercise test was started.

9. The standard Bruce protocol was followed and the subjects were asked to complete the walking till the second stage of Bruce protocol; if otherwise limited by symptoms of exhaustion or discomfort in which case they would be allowed to stop. This accounted for a 6 minute walk test with increased workload with the increase of gradient and speed of the treadmill.

10. At the end of the $2^{\text {nd }}$ stage, the treadmill was stopped and the subjects were allowed to rest.

11. Parameters recorded during the $1^{\text {st }}$ and the $2^{\text {nd }}$ stage of the treadmill stress test were:- Heart rate (resting \& target \& during stage $1 \&$ stage 2), blood pressure, METs \& Double Product. ECG waves were also monitored.

12. All the parameters were correlated with the BMI and the values recorded among the two study groups were studied and analyzed.

\section{Results \& observations:-}

1. The values recorded in both the groups correlated with the BMI. Subjects with higher BMI required greater energy to do the same exercise while those with lower BMI could perform the same exercise with lesser energy cost.

2. The resting heart rate was somewhat lower in the athletic subjects.

3. Changes in the Blood pressure and double product were almost similar in both the groups.

4. The subjects who played regular sports could work better in the treadmill with the same workload as compared to the ones leading sedentary lifestyle. This was evident by the fact that METs recorded seemed to be lower in the athletic group which meant that, the same workload was 'light work' for them as compared to those with sedentary lifestyle.

5. Thus athletic group had the ability to reach greater METs in due time as compared with the non athletic group who got fatigued early.

Analysis through tables and figures:-

Table 1:- Table showing the relation between BMI and METs recorded during TMT test.

\begin{tabular}{|l|l|l|l|l|l|l|}
\hline $\begin{array}{l}\text { BMI } \\
(\mathrm{Kg} / \mathrm{m} 2)\end{array}$ & $\begin{array}{l}\text { Underweight } \\
(<18.5)\end{array}$ & $\begin{array}{l}\text { Normal } \\
(18.5- \\
22.9)\end{array}$ & $\begin{array}{l}\text { Overweight } \\
(23-24.9)\end{array}$ & $\begin{array}{l}\text { Pre-obese } \\
(25-29.9)\end{array}$ & $\begin{array}{l}\text { Obese } \\
(>=30)\end{array}$ & P value \\
\hline No.of subjects & 4 & 18 & 11 & 7 & 0 & \\
\hline METs(Stage 1) & 4.40 & 4.62 & 4.74 & 4.91 & 0 & 0.02 \\
\cline { 1 - 2 } & 6.22 & 6.64 & 6.79 & 6.81 & 0 & (signf) \\
\hline
\end{tabular}

Table 2:- Table comparing the parameters recorded during TMT test between the two study groups:-

\begin{tabular}{|l|l|l|l|}
\hline $\begin{array}{l}\text { PARAMETERS } \\
\text { (average values) }\end{array}$ & $\begin{array}{l}\text { GROUP A } \\
\text { (Athletes) }\end{array}$ & $\begin{array}{l}\text { GROUP B } \\
\text { (Non-Athletes) }\end{array}$ & P value \\
\hline Resting HR (b/pm) & 68.2 & 77.6 & \multirow{2}{*}{$0.9(\mathrm{NS})$} \\
\hline HR(Stage 1) (b/pm) & 121.1 & 126.3 & \\
\hline HR(Stage 2) (b/min) & 142.4 & 148.9 & \\
\hline DP(Stage 1) & 17907.2 & 17375.6 & \\
\hline DP(Stage 2) & 22892.6 & 21528.4 & \\
\hline METs(Stage 1$)$ & 4.23 & 4.95 & \\
\hline METs(Stage 2) & 6.68 & 7.12 & \\
\hline
\end{tabular}


Figure 1:- Figure showing co-relation between BMI \& METS.

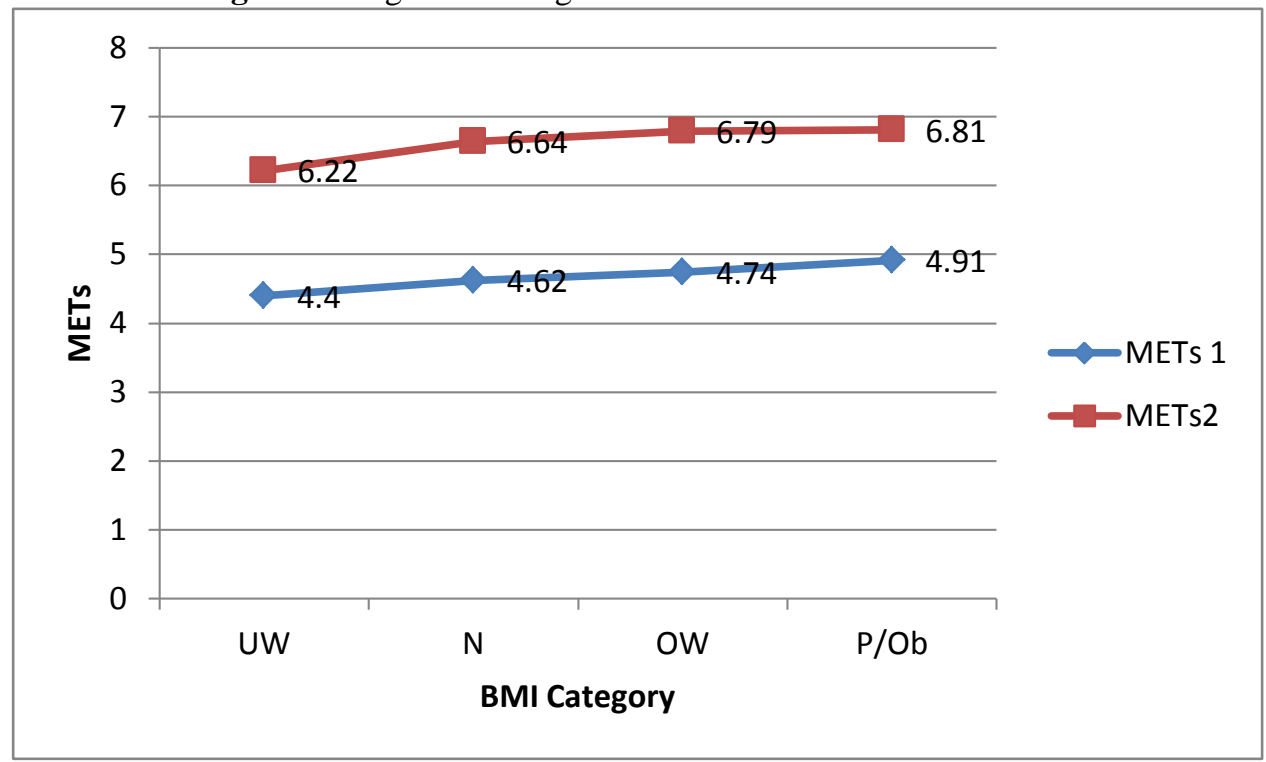

Figure 2:- Figure showing METs achieved by both the study groups.

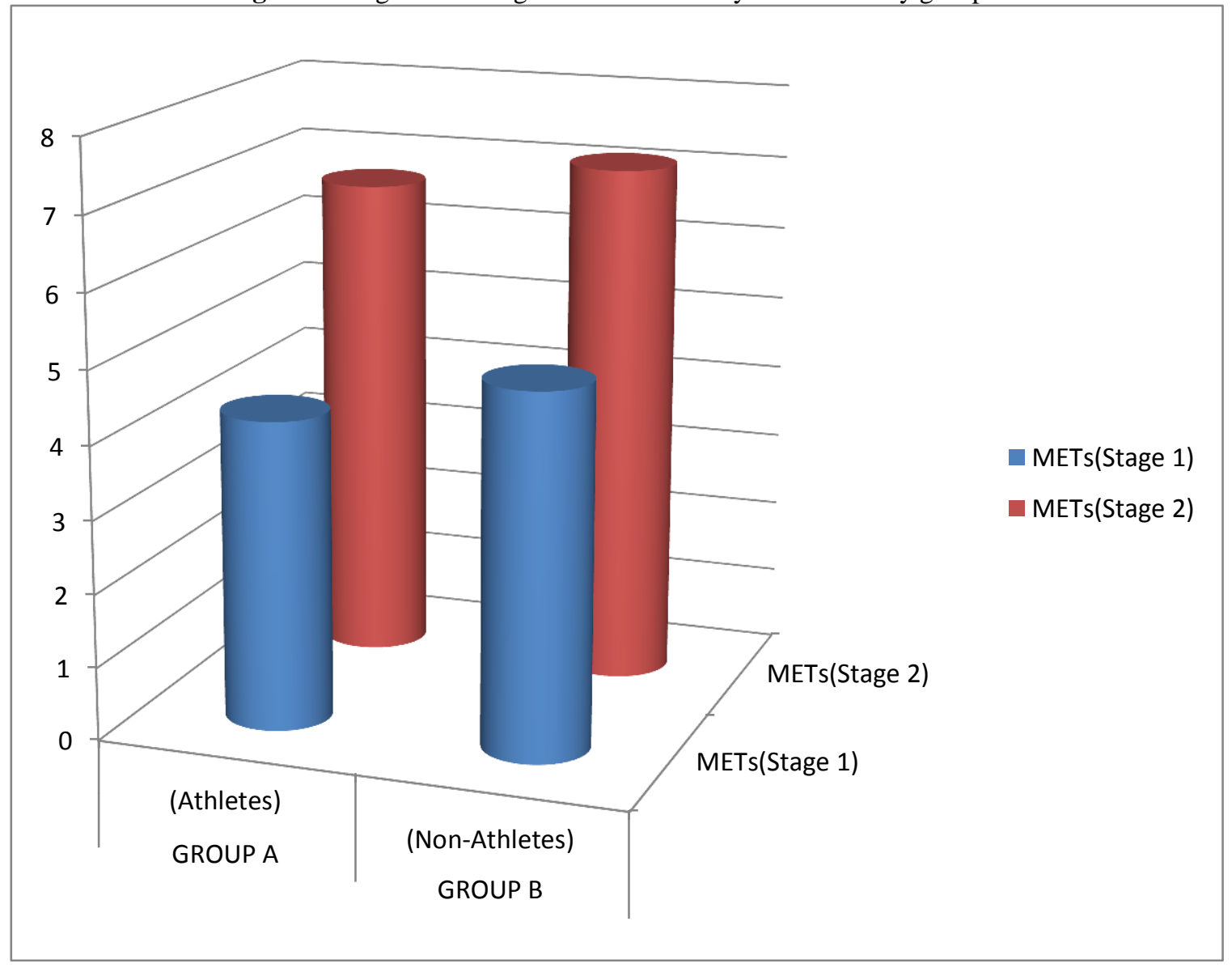


Figure 3:- Mason-Likar Lead placement taken from Welinder (2004).

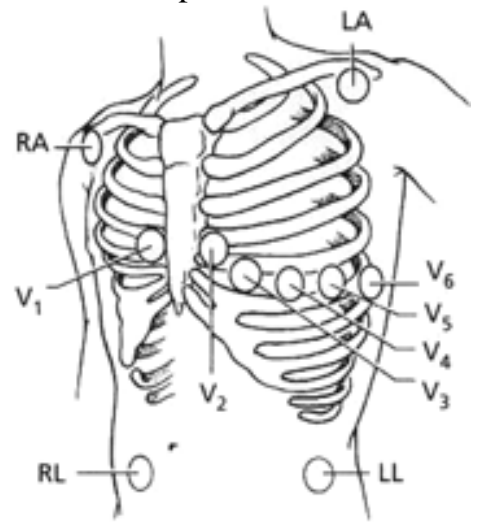

\section{Discussion:-}

1. M Jette et al. in 1990 stated that the MET concept provides a convenient method to describe the functional capacity or exercise tolerance of an individual as determined from progressive exercise testing.

2. G.Ramamruthy et al. in 1999 have said that maximal exercise capacity may be expressed in METs which corresponds to multiples of resting $\mathrm{O} 2$ consumption achieved during exercise. They have also said that double product or ratepressure product provides an indirect measurement of myocardial $\mathrm{O} 2$ requirement and higher product indicates better coronary perfusion.

3. Cureton and Sparling in 1980 said that maximal proportions of lean mass energy expenditure would be similar but subject with a greater fat mass would be unable to perform similar locomotion related maximal performance as the lean subject without an extra load.

4. Dr. LeWine reports that according to a study published in 2005 in the New England Journal of Medicine "Women whose exercise capacity [in METs] at the start of the study in 1992 was less than $85 \%$ of the predicted value for their age were twice as likely to have died over the next eight years compared with those who achieved $85 \%$ or better."

5. Dr. Howard LeWine, chief editor of Internet publishing, Harvard Health Publications, and clinical instructor of medicine at Harvard Medical School and Brigham and Women's Hospital says ${ }^{1}$ : "average healthy but nonathletic middle-aged men and women have peak exercise capacities in the range of 8 to 10 METs; marathon runners can have values as high as 18 to $24 . "$

6. T.A.Hargens et al in 2011 found that ,following Exercise trainning, maximal workload, and body composition variables all showed significant positive changes. The $\mathrm{VO}(2)$ at which the DPBP and VT occurred increased significantly from baseline to follow-up $(\mathrm{P}<0.001)$.

\section{Conclusion:-}

The results of the study thus provide some evidence to the fact that people having an athletic lifestyle have a better exercise capacity in terms of lower oxygen consumption at the same work load as seen by the value of the METs recorded. METs is an important measure of fitness. Target METs can be calculated using guidelines for appropriate age \& gender \& regular exercise can then be performed to improve the MET capacity which might increase our life expectancy and help in living a long healthy and happy life.

\section{References:-}

1. Ramamurthy Gita, Kerr Jamie E., Harsha David, Tavel Morton E. (1999), The Treadmill Test—Where To Stop and What Does It Mean?*, CHEST 1999; pp ( 115):1166-1169.

2. Cureton K. J., Sparling P. B. (1980). Distance running performance and metabolic responses to running in men and women with excess weight experimentally equated. Med. Sci. Sports Exerc. pp (12) , 288-294. 10.1249/00005768-198024000-00011.

3. Curran LS., Zhuang J., Droma T., and Moore LG. (1998). Superior exercise performance in lifelong Tibetan residents of 4,400 m compared with Tibetan residents of 3,658 m. Am J Phys Anthropol, pp (105):21-31 [PubMed].

4. Jetty M., Sidney K., Blumchen G. ( 1990 ), Metabolic Equivalents (METS) in Exercise Testing, Exercise Prescription, and Evaluation of Functional Capacity, Clin. Cardiol. pp (13) 555-565. 